\title{
Optimal Reinsurance via Dirac-Feynman Approach
}

\author{
Muhsin Tamturk ${ }^{1} \cdot$ Sergey Utev ${ }^{1}$
}

Received: 26 June 2017/ Revised: 16 May 2018 /

Accepted: 17 September 2018 / Published online: 4 October 2018

(c) The Author(s) 2018

\begin{abstract}
In this paper, the Dirac-Feynman path calculation approach is applied to analyse finite time ruin probability of a surplus process exposed to reinsurance by capital injections. Several reinsurance optimization problems on optimum insurance and reinsurance premium with respect to retention level are investigated and numerically illustrated. The retention level is chosen to decrease the finite time ruin probability and to guarantee that reinsurance premium covers an average of overall capital injections. All computations are based on Dirac-Feynman path calculation approach applied to the convolution type operators perturbed by Injection operator (shift type operator). In addition, the effect of the Injection operator on ruin probability is analysed.
\end{abstract}

Keywords Ruin probability · Reinsurance · Capital injection · Retention level ·

Dirac-Feynman approach

Mathematics Subject Classification (2010) 91B30 · 83C47 · 97K60

\section{Introduction}

The paper considers the application of the Quantum mechanic technique to compute the ruin probability of modified surplus process with reinsurance and the optimal reinsurance. Reinsurance is a risk sharing arrangement between a primary insurer and a reinsurer. There are different types of reinsurance agreements and various optimality approaches to reinsurance such as Castaner et al. (2013), Denuit and Vermandele (1998), Dickson and Waters (1996), Schmidli (2002), Zhou and Yuen (2012).

We consider the following reinsurance agreement motivated by Nie et al. (2011, 2015): the insured companies pay reinsurance premium in advance in order to get capital injections

Muhsin Tamturk

mt305@le.ac.uk

Sergey Utev

su35@le.ac.uk 
at times when the capital goes below a given retention level. Capital injection is an important topic in risk management, especially in case of unpredictable economic crisis or some natural disasters (Schmidli 2002; Dickson and Waters 2004; Eisenberg and Schmidli 2011).

Several optimal strategies with different distributions are discussed and numerically illustrated for the reinsurance agreement. All the methods have a main objective on the one hand to decrease the finite time ruin probability and on the other hand, to guarantee that reinsurance premium covers an average of overall capital injections. In addition, the 1st type of optimality is to find the optimal reinsurance premium and retention level to obtain the smallest ruin probability. The second type is to find the smallest premium rate against the retention level and a specific risk level.

In all our calculations, we apply the Dirac matrix approach (Baaquie 2007; Tamturk and Utev 2018). More exactly, all computations are based on the perturbed Dirac-Feynman path calculation approach applied to the convolution type operators such as the Dirac-Feynman operator, defined in Eqs. 4, 5 and 6, and Picard-Lefevre operator, defined in Eq. 1. The perturbation is defined as the Injection operator (shift type operator, introduced in Eq. 8) and associated with the particular reinsurance scheme.

In Section 2, following Tamturk and Utev (2018) we present several approaches to compute the finite time ruin probability. In Section 3, the modified ruin process and injection operator are introduced. In addition, a peculiar connection between the capital injection operator and the convolution operator is established and the effect of the injection operator is analysed in Section 3. The main numerical applications on optimization are stated in Sections 4 and 5. In Section 6, the difference between finite time reinsurance contracts and their infinite time counterparts is analysed.

\section{Preliminary}

Classical ruin process Asmussen and Albrecher (2010) (also referred to as surplus process) is defined by

$$
R_{t}=u+c t-S_{t}
$$

where $u$ is initial capital, $c$ is premium rate per unit time, $t$ is time, and $S_{t}=\sum_{i=1}^{N(t)} X_{i}$ is total claim amount determined by claim occurrences process $N_{t}$, homogeneous Poisson process with rate $\lambda$, and i.i.d. claim amounts $X_{i}$ which are also independent of Poisson process $N(t)$.

The ruin time and the finite time ruin probabilities associated with the surplus process $R_{t}$ are then defined by

$$
T=\min \left\{t>0, R_{t} \leq 0\right\}, P_{u}(T>t)=P\left(T>t \mid R_{0}=u\right) .
$$

Unlike infinite time, the finite time ruin probabilities do not admit simple expressions even in classical cases such as an exponential claim amount distributions.

Picard and Lefevre (1997) suggested a powerful approach based on the expansions via Appell polynomials, which also linked to the Seal-type formula (Rulliere and Loisel 2004; Lefevre and Loisel 2008). 
Lemma 1 (Picard-Lefevre). Fix non-negative integer $j$ such that $u+c t-j \geq 0$. Then,

$$
\begin{aligned}
& P_{u}\left(S_{t}=j, T>t\right)=e^{-\lambda t} A_{j}(t), \quad \text { where } \\
& A_{j}(t)=e_{j}(t) \text { for } 0 \leq j \leq u, A_{j}(t)=e_{j}\left(\frac{j-u}{c}\right) \frac{c x-n+u}{c x-j+u} e_{n-j}\left(x+\frac{u-j}{c}\right) \text { for } j>u, \\
& \text { and } e_{j}(t)=\sum_{k=0}^{j} \frac{(\lambda t)^{k}}{k !} P\left(X_{1}+\ldots+X_{k}=j\right) .
\end{aligned}
$$

The formula has been applied in different areas. In the recently published paper (Tamturk and Utev 2018), it's shown that Quantum Mechanic method and Picard- Lefevre method give very close results in computation of ruin probability for integer values.

General surplus process. Motivated by the Dirac-Feynman path integral approach, our starting point is to treat surplus process $R_{t}$ which admits the Hamiltonian representation

$$
P_{i, j}^{(\tau)}=P\left(R_{\tau}=j \mid R_{0}=i\right)=\left\langle i\left|e^{-\tau H}\right| j\right\rangle .
$$

By choosing a small time grid size $\epsilon$, we then derive the path calculation approximated version of the finite time ruin probability by

$$
\begin{gathered}
P_{u}\left(R_{t}=j, T>t\right)=(1+o(\varepsilon))\left(\sum_{x_{1}=1}\left\langle u|P| x_{1}\right\rangle\right. \\
\left.\ldots \sum_{x_{n-2}=1}\left\langle x_{n-2}|P| x_{n-1}\right\rangle\right)\left\langle x_{n-1}|P| j\right\rangle .
\end{gathered}
$$

where $P=P(\varepsilon)=e^{-\varepsilon H}$.

The operator $P$ does not have to be the transition operator. By conditioning on each claim, the Markov property and the Chapman-Kolmogoroff argument then imply the following lemma.

Lemma 2 Under assumption that 0 is an absorption state representing ruin probability, and the observing unit time $\varepsilon$ for bounded continuous function with $f(0)=0$

$$
E\left[f\left(R_{t}\right) I(T>t) \mid R_{0}=u\right]=(1+o(\varepsilon)) P^{\left[\frac{t}{\varepsilon}\right]} f(u)
$$

with Eq. 2, ruin and non-ruin probability can be computed by

$$
P_{u}(T>t)=(1+o(\varepsilon)) \sum_{j=1}^{\infty} P_{u, j \varepsilon}^{\left[\frac{t}{\varepsilon}\right]}, P_{u}(T \leq t)=(1+o(\varepsilon)) P_{u, 0}^{\left[\frac{t}{\varepsilon}\right]}
$$

where the error terms depend on the grid time size $\varepsilon$.

The choice of operator $P$ even for the same stochastic is not unique. For the classical surplus process, one way to state is to apply a quantum mechanics formalizm mentioned in (Tamturk and Utev 2018; Baaquie 2007)

$$
\begin{aligned}
P\left(x_{i} \rightarrow x_{i+1}\right) & =<x_{i}\left|e^{-\varepsilon H}\right| x_{i+1}>=\int_{0}^{2 \pi} \frac{d p}{2 \pi}<x_{i}\left|e^{-\varepsilon H}\right| p><p \mid x_{i+1}> \\
& =\frac{1}{2 \pi} \int_{0}^{2 \pi} e^{i p\left(x_{i}-x_{i+1}\right)+\varepsilon i c p-\varepsilon \sum_{j=1}^{\infty} \lambda_{j}\left(1-e^{-j i p}\right)} d p .
\end{aligned}
$$


On the other hand, it is possible to apply here a standard Panjer algorithm or the following straightforward approximations for $i+c>j$

$$
\begin{aligned}
P\left(R_{\varepsilon}=j \mid R_{0}=i\right) & =P\left(i+c-S_{\varepsilon}=j\right) \\
& =\lambda \varepsilon P\left(X_{1}=i+c-j\right)+O\left(\varepsilon^{2}\right) .
\end{aligned}
$$

Alternatively, we can apply the naive statistician approach and replace the $R_{t}$ process by the Gaussian process, which then results in the operator $P(t)=e^{-t H-V}$ where

$$
\begin{aligned}
P\left(x_{i} \rightarrow x_{i+1}\right) & =<x_{i}\left|e^{-\varepsilon H-V}\right| x_{i+1}> \\
& =\frac{1}{\sqrt{2 \pi \sigma_{\varepsilon}^{2}}} e^{\frac{-\left(x_{i+1}-\left(x_{i}+c \varepsilon-m \lambda \varepsilon\right)\right)^{2}}{2 \sigma_{\varepsilon}^{2}}} e^{-V\left(x_{i+1}\right)}
\end{aligned}
$$

where the potential $V\left(x_{i+1}\right)$ for the non-ruin probability is taken by

$$
V\left(x_{i+1}\right)= \begin{cases}0, & \text { if } x_{i+1}>0 \\ \infty, & \text { if } x_{i+1} \leq 0\end{cases}
$$

The variance is found by

$$
\sigma_{\varepsilon}^{2}=\operatorname{Var}\left(S_{\varepsilon}\right)=E\left[N_{\varepsilon}\right] \operatorname{Var}(X)+\operatorname{Var}\left(N_{\varepsilon}\right) E[X]^{2} .
$$

Notice that (5) defines an operator

$$
P f\left(x_{i}\right)=\sum_{x_{j}} P\left(x_{i} \rightarrow x_{j}\right) f\left(x_{j}\right) .
$$

refereed to as Dirac-Feynman operator (convolution type operator).

\section{Modified Ruin Model}

In this section, we will introduce the modified surplus process which incorporates the reinsurance by capital injections.

\subsection{Ruin Probabilities for the Modified Ruin Model}

As mentioned above, there are various types of reinsurance agreements provided by reinsurers. In this paper, we consider the reinsurance contract as discussed in Nie et al. (2011).

For this contract, the first insurance company has to pay the initial premium amount $z$ in advance to the second insurance company (referred to as reinsurer) that restores the surplus of first insurance company to a fixed retention level $(k)$ when the surplus process is below this retention level.

To make it more realistic, additionally, we assume the primary insurance company put an upper level for compensation of claims. Then, the aggregating claim amount with $h$ upper limit is defined by

$$
H\left(S_{t}\right)=\sum_{i=1}^{N_{t}} X_{i} I\left(X_{i} \leq h\right)+h I\left(X_{i}>h\right) .
$$

Although we are not dealing directly with model (7), we work with it in all numerical examples. The level $h$ depends on $m$, e.g. for $m=12, h=300$.

The modified surplus process is then defined by

$$
R_{t}=w+c t-H\left(S_{t}\right)+Y(t)
$$


where $w=u-z$ is the new initial capital after buying reinsurance and

$$
Y(t)=Y(w, k, t)=\sum_{i=1}^{\left[\frac{t}{\varepsilon}-1\right]} y_{i}
$$

is total injection amount up to time $t$, defined by the retention level $k$, grid time size $\varepsilon$ and exact initial capital $w$. Notice that under this reinsurance agreement the capital injections $y_{i}$ happen at times $i \varepsilon, i=1,2, \ldots$.

Now, let us introduce an Injection operator (shift type operator) with 0 absorption and $k$ retention level

$$
(K f)(x)=\left\{\begin{array}{l}
f(x), \quad \text { if } x \geq k \\
f(k), \text { if } 0<x<k \\
f(0), \quad \text { if } x \leq 0
\end{array}\right.
$$

Let $\bar{P}_{w}^{k}(T>t)$ and $\bar{P}_{w}^{k}(T \leq t)$ be non ruin and ruin probabilities of the modified surplus process. From Eq. 3 of Lemma 2 and Eq. 2, we derive

$$
\begin{aligned}
\bar{P}_{w}^{k}(T>t)=(1+o(1)) \sum_{x_{1}=1}<u|P K| x_{1}>\sum_{x_{2}=1}<x_{1}|P K| x_{2}> \\
\quad \cdots \sum_{x_{n}=1}<x_{n-1}|P| x_{n}> \\
=(1+o(1)) \sum_{j=1}(\underbrace{P K P K \ldots K}_{\left[\frac{t}{\varepsilon}-1\right] \text { times }} P)_{w, j \varepsilon}=(1+o(1)) \sum_{j=1}\left((P K)^{\left[\frac{t}{\varepsilon}-1\right]} P\right)_{w, j \varepsilon}
\end{aligned}
$$

and similarly

$$
\bar{P}_{w}^{k}(T \leq t)=(1+o(1))(\underbrace{P K P K \ldots K}_{\left[\frac{t}{\varepsilon}-1\right] \text { times }} P)_{w, 0}=(1+o(1))\left((P K)^{\left[\frac{t}{\varepsilon}-1\right]} P\right)_{w, 0} .
$$

In particular, we derive the following proposition.

Proposition 1 Under notation in above,

$$
\begin{aligned}
& \bar{P}_{w}^{k}(T>t)=(1+o(1)) \sum_{j=1}\left((P K)^{\left[\frac{t}{\varepsilon}-1\right]} P\right)_{w, j \varepsilon}, \\
& \bar{P}_{w}^{k}(T \leq t)=(1+o(1))\left((P K)^{\left[\frac{t}{\varepsilon}-1\right]} P\right)_{w, 0}
\end{aligned}
$$

where the error term depends on the grid time size $\varepsilon$.

\subsection{Effect of the Injection Operator for the Convolution Operator $P$}

Notice that $K^{n}=K$ and it is easier to work with

$$
P^{n} K^{n} P=P^{n} K P \text {. }
$$

Operators $(P K)^{n} P$ and $P^{n} K P$ are type of transition matrices. Note that in $(P K)^{n} P$, we apply injection $n$ times whereas in $P^{n} K P$, it is applied only once.

Unfortunately, the operators $K$ and $P$ are non-commutative in general, which clearly poses numerical complications. 
Stochastic comparison of $(P K)^{n} P$ and $P^{n} K P$ We first model the movement of the capital with initial capital $w$ via the operators $(P K)^{n} P$ and $P^{n} K P$ by a coupling construction. Let $R(n+1)$ and $\bar{R}(n+1)$ be capitals of surplus process at time $(n+1) \varepsilon$ via $(P K)^{n} P$ and $P^{n} K P$. By abuse of notation, here by $K$ we also denote a function

$$
K(x)=\left\{\begin{array}{l}
x \quad \text { if } x \geq k \\
k, \text { if } 0<x<k \\
0, \quad \text { if } x \leq 0 .
\end{array}\right.
$$

Notice that

$$
K(x) \geq x
$$

Let

$$
p_{i, j}=P\left(R_{0}+\xi=j \mid R_{0}=i\right)=P\left(\xi=j-i \mid R_{0}=i\right) \text { where } \xi=c-X_{1} .
$$

For $P^{n} K P$, the transition for one step $n=1$,

$$
w \longrightarrow w+\xi_{1} \longrightarrow K\left(w+\xi_{1}\right) \longrightarrow K\left(u+\xi_{1}\right)+\xi_{2} .
$$

For two steps $n=2$

$$
\begin{aligned}
w & \longrightarrow w+\xi_{1} \longrightarrow K\left(u-z+\xi_{1}\right) \longrightarrow K\left(u-z+\xi_{1}\right)+\xi_{2} \longrightarrow K\left(K\left(w+\xi_{1}\right)+\xi_{2}\right) \\
& \longrightarrow K\left(K\left(w+\xi_{1}\right)+\xi_{2}\right)+\xi_{3} .
\end{aligned}
$$

In general for $n$ steps, and $w>0$

$$
\begin{aligned}
& {\left[(P K)^{n} P\right]_{w, R(n+1)}=w \stackrel{(P K)^{n} P}{\longrightarrow} R(n+1)} \\
& \quad=K\left(K\left(\cdots K\left(w+\xi_{1}\right)+\xi_{2}\right)+\cdots\right)+\xi_{n+1} .
\end{aligned}
$$

Similar pattern for $P^{n} K P$ is given by

$$
\begin{aligned}
w & \stackrel{\overbrace{P}^{P^{n}}}{\longrightarrow} w+\xi_{1}+\xi_{2}+\cdots+\xi_{n} \stackrel{\overbrace{\longrightarrow}^{K}}{\longrightarrow} K\left(w+\xi_{1}+\xi_{2}+\cdots+\xi_{n}\right) \\
& \stackrel{\stackrel{P}{\longrightarrow}_{\longrightarrow}}{\longrightarrow} K\left(w+\xi_{1}+\xi_{2}+\cdots+\xi_{n}\right)+\xi_{n+1}
\end{aligned}
$$

which gives the capital at time $n+1$ when initial capital is $u$ and the grid size is 1 . Therefore,

$$
\left[P^{n} K P\right]_{w \bar{R}(n+1)}=w \stackrel{P^{n} K P}{\longrightarrow} \bar{R}(n+1)=K\left(w+\xi_{1}+\xi_{2}+\cdots+\xi_{n}\right)+\xi_{n+1}
$$

From Eqs. 10, 11 and 9, we derive the following coupling inequality

$$
\begin{aligned}
R(n+1) & =K\left(K\left(\cdots K\left(w+\xi_{1}\right)+\xi_{2}\right)+\cdots\right)+\xi_{m+1} \\
& \geq K\left(w+\xi_{1}+\xi_{2}+\cdots+\xi_{m}\right)+\xi_{m+1}=\bar{R}(n+1)
\end{aligned}
$$

which implies the following result.

Proposition 2 Under notation in above, for any integers $x \geq 1$ and $n \geq 0$,

$$
\sum_{j=x}\left((P K)^{n} P\right)_{w, j} \geq \sum_{j=x}\left(P^{n} K P\right)_{w, j}
$$

implying that the Ruin probabilities computed via $(P K)^{n} P$ are approximately smaller than corresponding Ruin probabilities computed via $P^{n} K P$.

By inspecting the proof, we see that the argument for the comparison holds provided the operator $P$ is non-decreasing, that is $P f$ maps non-decreasing functions to non-decreasing. 
Due to the stochastic comparison and Kamae et al. (1977) the Wasserstein distance can be computed via the Monte Carlo approach simultaneously

$$
d_{w}(R(t), \bar{R}(t))=E[R(t)-\bar{R}(t)] \approx \frac{1}{N} \sum_{j=1}^{N}\left[R^{j}(t)-\bar{R}^{j}(t)\right] .
$$

Numerical experiments were conducted for several groups of values of time $t=$ $30,45,60$ and retention levels $k=5,10,15,30,45$ with the initial capital being fixed $u=50$, premium rate $c=20$, the claim sizes have a discretized exponential distribution with claim mean 18, claim frequency 1 , and for upper barrier $h=500$.

In the criteria of selection of the parameters, since it is not feasible to check all possibilities, we consider firstly net profit condition $(c>\lambda m)$. Secondly, smaller claim frequency is preferred because it causes smaller errors.

According to the numerical results in Table 1, the ruin probabilities via $(P K)^{n} P$ are smaller than $P^{n} K P$ as expected and somewhat surprisingly close. The Wassertstein distance is relatively small when retention level $k$ is small. However, it is significantly increasing with the retention level $k$ and time $t$ while the ruin probabilities might still be close.

\subsection{Expectation of the Total Capital Injections Amount}

For the reinsurance to be reasonable for the reinsurance company, reinsurance cost $z$ is required to cover the average of the total injection amount, that is

$$
E[Y(u-z, k, t)]<z .
$$

We begin by stating the ready numerical formula for the expected total injection amount $E[Y(w, k, t)]$.

Proposition 3 Let 0 be the absorption level and $\varepsilon$ be the grid time size. Then, with $(P K)^{0}=$ I identity operator

$$
E[Y(w, k, t)]=\sum_{j=1}^{\left[\frac{t}{\varepsilon}-1\right]} \sum_{i=1}^{\frac{k}{\varepsilon}-1}(k-i \varepsilon)\left((P K)^{j-1} P\right)_{w, i \varepsilon} .
$$

Table 1 Ruin probability via $(P K)^{n} P$ and $P^{n} K P$

\begin{tabular}{lllll}
\hline Time & $\mathrm{k}$ & via $(P K)^{n} P$ & via $P^{n} K P$ & $d_{w}(R(t), \bar{R}(t))$ \\
\hline 30 & 5 & 0.4513 & 0.4527 & 0.1588 \\
30 & 10 & 0.4463 & 0.4526 & 0.7519 \\
30 & 15 & 0.4084 & 0.4524 & 5.6104 \\
45 & 5 & 0.4837 & 0.4851 & 0.1745 \\
45 & 15 & 0.4700 & 0.4850 & 2.0119 \\
45 & 30 & 0.4206 & 0.4849 & 9.1205 \\
60 & 5 & 0.5005 & 0.5018 & 0.1777 \\
60 & 30 & 0.4376 & 0.5017 & 9.2851 \\
60 & 45 & 0.3602 & 0.5015 & 21.6717 \\
\hline
\end{tabular}


Proof For simplicity, let $\varepsilon=1$. Let us fix time $j$ and the corresponding capital $R_{j}=i$. Notice that if $0<i<k$ then at time $j$ we will add the injection amount $y_{j}=k-i$.

Clearly, the expectation of capital injections paid by reinsurer at time $j$ is defined by

$$
E\left[y_{j}\right]=\sum_{i=1}^{k-1}(k-i)(P K P K \ldots K P)_{w, i}=\left((P K)^{j-1} P\right)_{w, i} .
$$

Then, the total injection amount is found by combining all the added amounts over times $j=1, \ldots, t-1$ and possible injection capitals $i=1, \ldots, k-1$.

$$
E[Y(w, k, t)]=\sum_{j=1}^{t-1} E\left[y_{j}\right]=\sum_{j=1}^{t-1} \sum_{i=1}^{k-1}(k-i)\left((P K)^{j-1} P\right)_{w, i} .
$$

\section{Optimization in Gaussian Hamiltonian}

\subsection{Optimization of Reinsurance Cost z}

In this part, finite time ruin probability of modified surplus process and expected total injection amount are numerically computed using the methods above. The results are analysed for the time $t=20$, initial capital $u=20$, premium rate $c=14$, claim frequency $\lambda=1$, claim mean $m=12, \operatorname{var}(X)=144$ and $h=\infty$.

From Lemma 2, we find that the finite time ruin without reinsurance is equal to

$$
P_{20}(T \leq 20)=0.6110 .
$$

In addition, for simplicity we analyse the modified surplus process for reinsurance costs $z=\{1,2, \ldots, 10\}$ and retention levels $k=\{5,6, \ldots, 10\}$ (Table 2).

Our aim is to minimise the finite time ruin probability and corresponding reinsurance premium $z$, that is to find

$$
\min \left\{\bar{P}_{u-z}^{k}(T \leq t): z>E[Y(u-z, k, t)] \text { and } \bar{P}_{u-z}^{k}(T \leq t)<P_{u}(T \leq t)\right\} .
$$

Table 2 Ruin probability of the modified surplus process with respect to $z$ and $k$

\begin{tabular}{lllllll}
\hline & $\bar{P}_{w}^{k}(T \leq 20)$ & & & \\
\cline { 2 - 6 } & $\mathrm{k}=5$ & $\mathrm{k}=6$ & $\mathrm{k}=7$ & $\mathrm{k}=8$ & $\mathrm{k}=9$ & $\mathrm{k}=10$ \\
\hline $\mathrm{z}=1$ & 0.616 & 0.612 & 0.6071 & 0.6011 & 0.594 & 0.5859 \\
$\mathrm{z}=2$ & 0.6285 & 0.6244 & 0.6195 & 0.6135 & 0.6064 & 0.5983 \\
$\mathrm{z}=3$ & 0.6409 & 0.6369 & 0.6319 & 0.6259 & 0.6189 & 0.6107 \\
$\mathrm{z}=4$ & 0.6535 & 0.6495 & 0.6445 & 0.6385 & 0.6315 & 0.6233 \\
$\mathrm{z}=5$ & 0.666 & 0.662 & 0.6571 & 0.6511 & 0.6441 & 0.636 \\
$\mathrm{z}=6$ & 0.6786 & 0.6746 & 0.6696 & 0.6637 & 0.6568 & 0.6487 \\
$\mathrm{z}=7$ & 0.6911 & 0.6871 & 0.6822 & 0.6764 & 0.6695 & 0.6615 \\
$\mathrm{z}=8$ & 0.7036 & 0.6997 & 0.6948 & 0.689 & 0.6822 & 0.6743 \\
$\mathrm{z}=9$ & 0.716 & 0.7122 & 0.7074 & 0.7016 & 0.6949 & 0.6871 \\
$\mathrm{z}=10$ & 0.7284 & 0.7246 & 0.7198 & 0.7141 & 0.7075 & 0.6999 \\
\hline
\end{tabular}


According to numerical results, it is clear that the reinsurance is appropriate for several values of $k$ and $z$. However, we choose the value of reinsurance cost $z=3$ and the retention level $k=10$ because it gives the smallest ruin probability (0.6107) under the conditions that $z>E[Y(u-z, k, t)]$ and $\left.\bar{P}_{u-z}^{k}(T \leq t)<P_{u}(T \leq t)\right\}$.

\subsection{Optimization of the Premium Rate c}

A numerical example to find the minimal premium $c$ is considered. We find it via optimization of the retention level $k$ with respect to specific risk level $L$. Smaller insurance premium plays an important role in competitive sector to increase number of customers which effects the income of insurance companies. In this case,

$$
\begin{aligned}
& u=20, \lambda=1, m=12, t=40, \operatorname{Var}(X)=\sigma_{X}^{2}=144, \\
& z=5, k=\{5,6, \ldots, 10\}, h=\infty \text { and } c=\{10,11,12,13,14,15\} .
\end{aligned}
$$

The ruin probabilities now depends on the premium rate $c$ written as $P_{u}(T \leq t \mid c)$ and $\bar{P}_{w}^{k}(T \leq t \mid c)$. The goal is to find minimal $c$ such that there exists $k$ satisfying

$$
L \leq \bar{P}_{15}^{k}(T \leq 40 \mid c) \leq P_{20}(T \leq 40 \mid c) \text { and } z>E(Y)
$$

Again, the optimal $c$ depends on the level $L$ and $k$. For $L=0.8$ and $k=8$, the minimal premium rate is attained for $c=12$ corresponding to $\bar{P}_{15}^{8}(T \leq 40 \mid c)=0.8045$.

\section{Optimization in Compound Poisson Hamiltonian for Claim Sizes Distributed Exponentially}

\subsection{Optimization of Reinsurance Cost z}

Similar to Section 4.1, the ruin probability and total injection amount are analysed in case that claims are exponentially distributed for initial capital $u=20$, premium rate $c=14$, claim frequency $\lambda=1$, claim mean $m=12$, time $t=20$ and $h=\infty$.

In case of no reinsurance, the ruin probability is

$$
P_{20}(T \leq 20)=0.5438 .
$$

According to numerical results, optimum reinsurance where the reinsurance agreement is reasonable is attained by $k=8$ and $z=1$ because

$$
\min \left\{\bar{P}_{u-z}^{k}(T \leq t)\right\}=\bar{P}_{19}^{8}(T \leq 20)=0.5385
$$

providing that

$$
z>E[Y(u-z, k, t)] \text { and } \bar{P}_{u-z}^{k}(T \leq t)<P_{u}(T \leq t) .
$$

\subsection{Optimization of the Premium Rate c}

If the ruin probability and the expected total injection amount for exponential claim distribution are taken into consideration for $u=20, z=5, t=40, \lambda=1$ and $m=12$.

Table 3 shows that the ruin probabilities under reinsurance agreement are higher than the case without reinsurance. Therefore, reinsurance agreement is not reasonable for the values. 
Table 3 Ruin probability with respect to $k$ and $c$

$$
P_{20}(T \leq 40) \quad \bar{P}_{15}^{k}(T \leq 40)
$$

(No reinsurance)

\begin{tabular}{llllllll}
\cline { 3 - 7 } & & $\mathrm{k}=5$ & $\mathrm{k}=6$ & $\mathrm{k}=7$ & $\mathrm{k}=8$ & $\mathrm{k}=9$ & $\mathrm{k}=10$ \\
\hline $\mathrm{c}=10$ & 0.9129 & 0.9256 & 0.9245 & 0.9231 & 0.9214 & 0.9194 & 0.9171 \\
$\mathrm{c}=11$ & 0.8537 & 0.8733 & 0.8718 & 0.87 & 0.8678 & 0.8652 & 0.8622 \\
$\mathrm{c}=12$ & 0.781 & 0.8068 & 0.8051 & 0.8029 & 0.8003 & 0.7973 & 0.7938 \\
$\mathrm{c}=13$ & 0.6988 & 0.7313 & 0.7294 & 0.727 & 0.7242 & 0.7209 & 0.7171 \\
$\mathrm{c}=14$ & 0.6148 & 0.6525 & 0.6505 & 0.648 & 0.6451 & 0.6418 & 0.638 \\
$\mathrm{c}=15$ & 0.5344 & 0.5767 & 0.5748 & 0.5724 & 0.5696 & 0.5664 & 0.5628 \\
\hline
\end{tabular}

\section{Comparison of Finite and Infinite Time Methods}

In this section, we compare our finite time ruin probabilities with the infinite time ruin probabilities of modified surplus processes. In finite time, all computation are produced via the quantum path calculation approach stated in Lemma 2 (and Eqs. 4, 5 and 6).

Recall that $P_{u}(T<\infty)$ and $\bar{P}_{u}^{k}(T<\infty)$ denote the ultimate ruin probabilities for the classical and modified surplus processes with retention level $k$, respectively.

To compare finite time ruin probability with infinite time ruin probability, the following fact will be applied

$$
\frac{P(T \leq M)}{P(T \leq L)} \rightarrow 1(\text { as } L, M \rightarrow \infty), \quad \text { and so } \quad \frac{P(T<\infty)}{P(T \leq L)} \rightarrow 1,(\text { as } L \rightarrow \infty) .
$$

We apply infinite time ruin probabilities of modified surplus processes derived in Nie et al. (2011):

$$
\bar{P}_{w}^{k}(T<\infty)=P_{w-k}(T<\infty)-G(w-k, k) \frac{1-P_{0}(T<\infty)}{1-G(0, k)}
$$

where $u-z>k, G(x, k)=P_{x}(T<\infty)\left(1-e^{-\alpha k}\right)$ and the claim size has an exponential distribution with rate $\alpha$. Moreover, the expectation of the total injection amount is defined by

$$
E[Y(w, k)]=\int_{0}^{k} y g(w-k, y) d y+E[Y(k, k)] G(w-k, k)
$$

where $g(w-k, y)=P_{w-k}(T<\infty) \alpha e^{-\alpha y}$.

Our finite time method introduced in Eqs. 2 and 1 with discretized, truncated exponential claim sizes will be compared with the infinite time formula above. Remind, that the ultimate ruin probability of classical surplus process is defined by (e.g. Nie et al. 2011)

$$
P_{u}(T<\infty)=\frac{\lambda m}{c} e^{-\left(\frac{1}{m}-\frac{\lambda}{c}\right) u} .
$$

In the following two Tables 4 and 5, the ruin probabilities for normal and modified surplus processes under capital injections are compared for our finite approach with the infinite tie approach as in Eq. 13. In both tables, we let $R_{0}=u-z=w$ in case of reinsurance while $R_{0}=u$ without reinsurance.

Ruin probability and expected total injection amount in the finite and infinite time methods for the initial capital $u=20$, insurance premium $c=1$, claim frequency $\lambda=0.03$, 
Table 4 Ruin probabilities and total injection amount

\begin{tabular}{|c|c|c|c|c|c|}
\hline \multirow[b]{2}{*}{ Reinsurance premium } & \multirow[b]{2}{*}{$\mathrm{w}=\mathrm{u}-\mathrm{z}$} & \multicolumn{2}{|c|}{ Infinite time method } & \multicolumn{2}{|c|}{ Finite time method } \\
\hline & & $\bar{P}_{w}^{k}(T<\infty)$ & $E[Y(w, k)]$ & $\bar{P}_{w}^{k}(T \leq 1400)$ & $E[Y(w, k, 1400)]$ \\
\hline $\mathrm{z}=1$ & 19 & 0.8437 & 0.3719 & 0.8374 & 0.2746 \\
\hline $\mathrm{z}=2$ & 18 & 0.8465 & 0.3731 & 0.8404 & 0.2758 \\
\hline $\mathrm{z}=3$ & 17 & 0.8493 & 0.3744 & 0.8435 & 0.2771 \\
\hline $\mathrm{z}=4$ & 16 & 0.8521 & 0.3756 & 0.8466 & 0.2784 \\
\hline $\mathrm{z}=5$ & 15 & 0.855 & 0.3769 & 0.8496 & 0.2797 \\
\hline $\mathrm{z}=6$ & 14 & 0.8578 & 0.3781 & 0.8527 & 0.281 \\
\hline $\mathrm{z}=7$ & 13 & 0.8607 & 0.3794 & 0.8558 & 0.2823 \\
\hline $\mathrm{z}=8$ & 12 & 0.8636 & 0.3807 & 0.8589 & 0.2836 \\
\hline $\mathrm{z}=9$ & 11 & 0.8665 & 0.3819 & 0.862 & 0.2849 \\
\hline $\mathrm{z}=10$ & 10 & 0.8694 & 0.3832 & 0.8652 & 0.2862 \\
\hline
\end{tabular}

claim mean $m=30$, the retention level $k=5$ are listed with respect to various reinsurance premium $z=1,2, \ldots, 10$ in Table 4 .

For the same values but different retention level $(\mathrm{k}=10)$, the results are listed in Table 5

As seen from Tables 4 and 5, Infinite time method gives larger ruin probability and expected injection amount compared to the finite time method. As expected, an increase in the retention level $k$ cause a decrease in ruin probability with larger expected injection amount.

Now, let's observe ruin probabilities of surplus process with and without reinsurance for both methods by keeping the initial capital $w=u-z$ being fixed.

Ruin probabilities of modified surplus process and the expected total injection amount for $w=10, c=1, \lambda=0.01, m=90$ are listed in Table 6 .

Similarly, for $w=40$ with same parameters $c, \lambda, m$, the results with various retention level $k=[5,10,15, \ldots, 40]$ will be in the following Table 7 .

Table 5 Ruin probabilities and total injection amount

\begin{tabular}{|c|c|c|c|c|c|}
\hline \multirow[b]{2}{*}{ Reinsurance premium } & \multirow[b]{2}{*}{$\mathrm{w}=\mathrm{u}-\mathrm{z}$} & \multicolumn{2}{|c|}{ Infinite time method } & \multicolumn{2}{|c|}{ Finite time method } \\
\hline & & $\bar{P}_{w}^{k}(T<\infty)$ & $E[Y(w, k)]$ & $\bar{P}_{w}^{k}(T \leq 1400)$ & $E[Y(w, k, 1400)]$ \\
\hline $\mathrm{z}=1$ & 19 & 0.8402 & 1.5697 & 0.8339 & 1.301 \\
\hline $\mathrm{z}=2$ & 18 & 0.843 & 1.575 & 0.8369 & 1.307 \\
\hline$z=3$ & 17 & 0.8458 & 1.5802 & 0.84 & 1.3131 \\
\hline$z=4$ & 16 & 0.8486 & 1.5855 & 0.843 & 1.3192 \\
\hline $\mathrm{z}=5$ & 15 & 0.8514 & 1.5908 & 0.8461 & 1.3253 \\
\hline$z=6$ & 14 & 0.8543 & 1.5961 & 0.8492 & 1.3315 \\
\hline$z=7$ & 13 & 0.8571 & 1.6014 & 0.8522 & 1.3377 \\
\hline$z=8$ & 12 & 0.86 & 1.6068 & 0.8553 & 1.3439 \\
\hline $\mathrm{z}=9$ & 11 & 0.8629 & 1.6122 & 0.8584 & 1.3501 \\
\hline $\mathrm{z}=10$ & 10 & 0.8657 & 1.6175 & 0.8616 & 1.3563 \\
\hline
\end{tabular}


Table 6 Ruin probabilities and total injection amount

\begin{tabular}{|c|c|c|c|c|}
\hline \multirow[b]{2}{*}{ Retention level } & \multicolumn{2}{|c|}{ Infinite time method } & \multicolumn{2}{|c|}{ Finite time method } \\
\hline & $\bar{P}_{w}^{k}(T<\infty)$ & $E[Y(w, k)]$ & $\bar{P}_{w}^{k}(T \leq 1400)$ & $E[Y(w, k, 1400)]$ \\
\hline $\mathrm{k}=1$ & 0.8901 & 0.005 & 0.8073 & 0 \\
\hline $\mathrm{k}=2$ & 0.89 & 0.0199 & 0.8073 & 0.009 \\
\hline $\mathrm{k}=3$ & 0.89 & 0.045 & 0.8073 & 0.027 \\
\hline $\mathrm{k}=4$ & 0.89 & 0.0803 & 0.8073 & 0.0542 \\
\hline $\mathrm{k}=5$ & 0.8899 & 0.1259 & 0.8072 & 0.0906 \\
\hline $\mathrm{k}=6$ & 0.8899 & 0.182 & 0.8071 & 0.1364 \\
\hline $\mathrm{k}=7$ & 0.8898 & 0.2486 & 0.807 & 0.1916 \\
\hline $\mathrm{k}=8$ & 0.8897 & 0.3259 & 0.8069 & 0.2564 \\
\hline $\mathrm{k}=9$ & 0.8896 & 0.414 & 0.8067 & 0.3309 \\
\hline $\mathrm{k}=10$ & 0.8895 & 0.513 & 0.8066 & 0.4151 \\
\hline
\end{tabular}

Discussion The tables above show that the reinsurance is not always appropriate (as also discussed in Nie et al. 2011). This is because the conditions in Eq. 12 are not satisfied; in addition, higher reinsurance cost $z$ does not imply smaller ruin probability in general.

Notice that the finite time methods even work when the ultimate ruin probability is 1, i.e. the net profit condition is violated $(c \ngtr \lambda m)$.

The advantage of the Dirac-Feynman approach is that we do not need to choose a particular Hamiltonian operator. In addition, together with the discrete time and state approximation it allows to avoid the need to obtain the joint distribution of the time to ruin and the deficit at ruin in order to obtain quantities of interest. Therefore, it makes the method more flexible. The disadvantage of the method is that the computation involved takes more time for the Levy processes.

As numerically illustrated in the previous paper (Tamturk and Utev 2018), the Quantum mechanics method for the Compound Poisson Hamiltonian, the Picard-Lefevre formula and Markov Chain methods give very close results, so by replacing the computation for the finite time ruin probability the similar results in optimization cases are expected. In the Gaussian

Table 7 Ruin probabilities and total injection amount

\begin{tabular}{|c|c|c|c|c|}
\hline \multirow[b]{2}{*}{ Retention level } & \multicolumn{2}{|c|}{ Infinite time method } & \multicolumn{2}{|c|}{ Finite time method } \\
\hline & $\bar{P}_{w}^{k}(T<\infty)$ & $E[Y(w, k)]$ & $\bar{P}_{w}^{k}(T \leq 1400)$ & $E[Y(w, k, 1400)]$ \\
\hline $\mathrm{k}=5$ & 0.8512 & 0.1218 & 0.7519 & 0.0843 \\
\hline $\mathrm{k}=10$ & 0.8509 & 0.4962 & 0.7513 & 0.3861 \\
\hline $\mathrm{k}=15$ & 0.8503 & 1.137 & 0.7503 & 0.917 \\
\hline $\mathrm{k}=20$ & 0.8494 & 2.0581 & 0.7488 & 1.6881 \\
\hline $\mathrm{k}=25$ & 0.8482 & 3.274 & 0.7468 & 2.7104 \\
\hline $\mathrm{k}=30$ & 0.8467 & 4.799 & 0.7444 & 3.9944 \\
\hline $\mathrm{k}=35$ & 0.845 & 6.6478 & 0.7415 & 5.5502 \\
\hline $\mathrm{k}=40$ & 0.8523 & 8.8351 & 0.738 & 7.3873 \\
\hline
\end{tabular}


Hamiltonian case, the results are slightly different due to the poor Gaussian approximation for the small initial capital or small time $t$.

Open Access This article is distributed under the terms of the Creative Commons Attribution 4.0 International License (http://creativecommons.org/licenses/by/4.0/), which permits unrestricted use, distribution, and reproduction in any medium, provided you give appropriate credit to the original author(s) and the source, provide a link to the Creative Commons license, and indicate if changes were made.

\section{References}

Asmussen S, Albrecher H (2010) Ruin probabilities. World Scientific, Singapore

Baaquie BE (2007) Quantum finance: Path integrals and Hamiltonians for options and interest rates. Cambridge University press, Cambridge

Castaner A, Claramunt MM, Lefevre C (2013) Survival probabilities in bivariate risk models, with application to reinsurance. Insur: Math Econ 53:632-642

Denuit M, Vermandele C (1998) Optimal reinsurance and stop-loss order. Insur: Math Econ 22:229-233

Dickson DC, Waters HR (1996) Reinsurance and ruin. Insur: Math Econ 19:61-80

Dickson DC, Waters HR (2004) Some optimal dividends problems. Astin Bullet 34:49-74

Eisenberg J, Schmidli H (2011) Minimising expected discounted capital injections by reinsurance in a classical risk model. Scand Actuar J 2011(3):155-176

Kamae T, Krengel U, O’Brien GL (1977) Stochastic inequalities on partially ordered spaces. Ann Probab 5(6):899-912

Lefevre C, Loisel S (2008) On finite-time ruin probabilities for classical risk models. Scand Actuar J 2008(1):41-60

Picard P, Lefevre C (1997) The probability of ruin in finite time with discrete claim size distribution. Scand Actuar J 1997(1):58-69

Nie C, Dickson DC, Li S (2011) Minimizing the ruin probability through capital injections. Ann Actuar Sci 5:195-209

Nie C, Dickson DC, Li S (2015) The finite time ruin probability in a risk model with capital injections. Scandinavian Actuarial Journal 2015(4):301-318

Rulliere D, Loisel S (2004) Another look at the Picard-Lefevre formula for finite-time ruin probabilities. Insur: Math Econ 35:187-203

Schmidli H (2002) On minimizing the ruin probability by investment and reinsurance. Ann Appl Probab 12:890-907

Tamturk M, Utev S (2018) Ruin probability via Quantum Mechanics Approach. Insur: Math Econ 79:69-74

Zhou M, Yuen KC (2012) Optimal reinsurance and dividend for a diffusion model with capital injection: Variance premium principle. Econ Model 29:198-207 